\title{
Stereo PIV measurements of oscillatory plasma forcing in the cross-plane of a channel flow
}

\author{
M. T. Hehner ${ }^{1 *}$, L. H. von Deyn ${ }^{1}$, J. Serpieri ${ }^{1}$, S. Pasch ${ }^{1}$, T. Reinheimer ${ }^{2}$, \\ D. Gatti ${ }^{1}$, B. Frohnapfel ${ }^{1}$, J. Kriegseis ${ }^{1}$ \\ ${ }^{1}$ Institute of Fluids Mechanics (ISTM), Karlsruhe Institute of Technology (KIT), Germany \\ ${ }^{2}$ Institute for Applied Materials (IAM), Karlsruhe Institute of Technology (KIT), Germany \\ *marc.hehner@kit.edu
}

\begin{abstract}
The present work describes an experimental investigation that applies stereo particle image velocimetry in a cross-plane of a turbulent channel flow that is additionally perturbed by spanwise oscillatory body forces, induced by a plasma actuator and designed to mimic the effect of spanwise wall oscillations. The experiment is aimed at retrieving the forcing-correlated scales and the turbulent flow stochastic fluctuations for the measured cross-plane. The first are macroscopic scales and require a larger investigation domain while the latter benefit of a higher resolution. Furthermore, the extended flow-field dynamic range posed a challenge on the experiment design, finally leading to an optimal tradeoff. The results of the unactuated flow compare well to the direct numerical simulations of Hoyas and Jiménez (2008), while the actuated case demonstrates strong near-wall momentum addition and spanwise modulation of the streamwise flow component.
\end{abstract}

\section{Introduction}

Control of wall-bounded flows is a broad topic at which many numerical investigations were focused on friction-drag reduction in turbulent channel flows (see review Quadrio (2011)) that can be achieved by streamwise traveling waves (StTWs) of spanwise wall velocity (Quadrio and Ricco (2011), Gatti and Quadrio (2016)). Experimental realization of StTWs typically require complex devices with actively moving components (Choi et al. (1998), Auteri et al. (2010)). Whereas implementations of StTWs without any moving parts are currently being investigated by means of plasma actuators (Jukes et al. (2006), Hehner et al. $(2019,2020))$, a particular class of actuator which can be flush-mounted to the surface and produces a mainly wall-parallel body force (see reviews Benard and Moreau (2014), Kotsonis (2015), Kriegseis et al. (2016)). Therefore, the objective of this work is to provide an advanced measurement system for which underlying mechanisms of a manipulated/controlled flow and the inherent effects on friction drag can be studied in a channel and compared to an uncontrolled flow. The means of choice, as shown in figure 1, is a stereo particle image velocimetry (sPIV) setup which captures a cross-plane of the channel flow $(y, z)$ (see also Kriegseis et al. (2015) for this type of setup). This setup allows reconstruction of spanwise (w), wall-normal $(v)$ and streamwise $(u)$ velocity components. Hence, the present work particularly revolves around both qualification of the sPIV setup in an uncontrolled fully-developed turbulent channel flow and topological interplay effects between oscillatory plasma discharges and external flow.

\section{Experimental procedure}

The windtunnel test section is a channel with a length, width and height of $4000 \mathrm{~mm} \times 300 \mathrm{~mm} \times 25.2 \mathrm{~mm}$ (channel semi height $h=12.6 \mathrm{~mm}$ ), respectively, and the measurement plane of the sPIV setup was arranged $100 \mathrm{~mm}$ upstream of the channel exit. Note that the coordinate system $(x, y, z)$ and corresponding flow components $(u, v, w)$ are indicated in the inset of figure 1 (upper left) which also shows the operating plasma-actuator array at the channel wall. The sPIV setup was run in double-frame mode and image acquisition was carried out by two Photron FASTCAM SA4 high-speed cameras that were placed at the left and 
right side (angles of about 27 degrees) of the channel exit. The seeding particles (Di-Ethyl-Hexyl-Sebacate) were illuminated by a Quantronix Darwin-Duo Nd:YLF laser (pulse width: $0.12 \mu \mathrm{s}$ ) that accesses the channel test section through a convex lens window, used to parallelize the light sheet to the walls, thus minimizing reflection issues from the walls. A central synchronizer control unit (ILA_5150 GmbH) was used to drive the laser pulses, camera exposures and the high-voltage transformers for the plasma discharges. Accordingly, for the plasma-actuated flow the sPIV measurements were phase-locked, in order to resolve 24 bins of the plasma oscillation cycle.

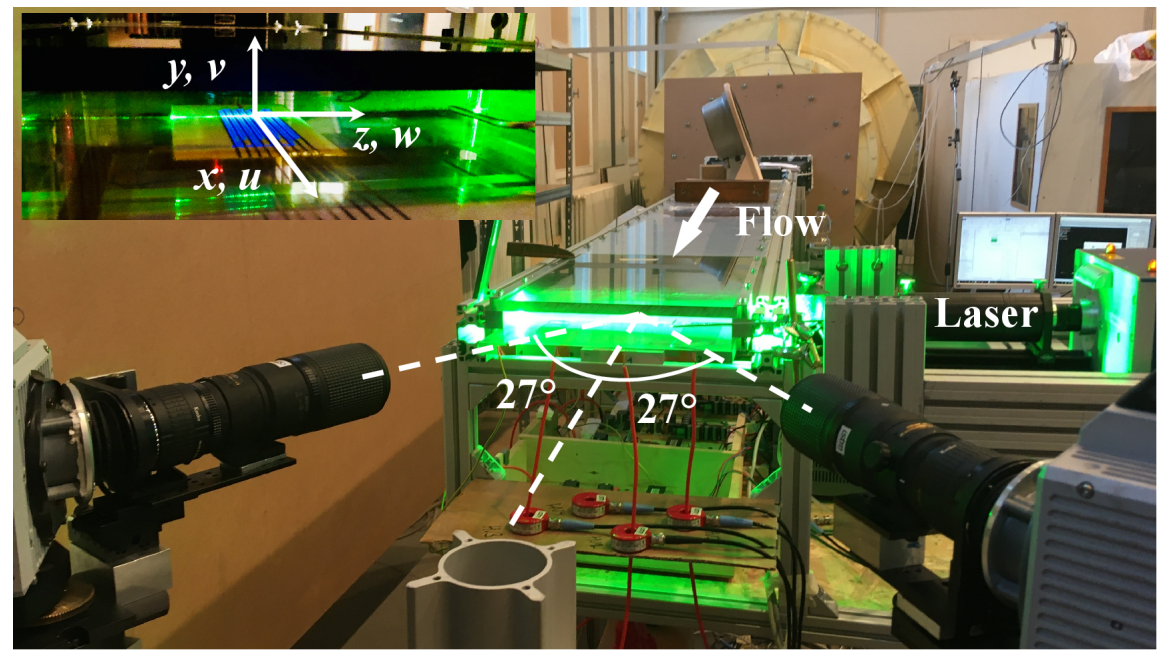

Figure 1: Experimental sPIV setup in the cross-plane of a fully-developed turbulent channel flow; top left inset shows the corresponding coordinate system and the investigated plasma-actuator array.

As a peculiarity of this sPIV framework, due to the cross-plane measurement of the main flow, there is a difference of at least an order of magnitude between the acquired in- and out-of-plane flow components. For a light-sheet thickness of about $30 \mathrm{px}$, the maximum particle out-of-plane displacements were locked at $\Delta x=10 \mathrm{px}$. The cameras were operated (sampling frequencies 500 to $1400 \mathrm{~Hz}$ ) at full sensor size $\left(1024 \times 1024 \mathrm{px}^{2}, 12\right.$ bits), spanning a field of view (FOV) of $16 \times 14 \mathrm{~mm}^{2}$ in width and height, respectively. In order to gain a spatial resolution of $r e s \approx 60 \mathrm{px} / \mathrm{mm}$ and to prevent the cameras from blocking the channel exit at the same time, both cameras were each equipped with a teleconverter (magnification factor 2) and a Nikon Nikkor $200 \mathrm{~mm}$ lens $\left(f_{\#}=11\right)$. Hence, the pulse delay $\mathrm{d} t$ was

$$
\mathrm{d} t=\frac{\Delta x}{r e s U_{\mathrm{cl}}} 10^{-3} \mathrm{~s}
$$

where $U_{\mathrm{cl}}$ is the (out-of-plane) center-line velocity that was continuously measured with a Prandtl tube near the channel exit, in order to determine the flow conditions (see sec. 3.1 for $U_{\mathrm{cl}}$ range). The FOV was located in the horizontal center of the channel (and of the actuator array, see also fig. 3) on the lower wall and imaged the area up to the semi height $h$ of the channel. Given the extended dynamic range and the underlying stochastic field, about 14,000 vector fields were acquired to guarantee minimal measurement random error and converged flow. Data processing was performed with PIVTECs PIVview software (version 3.8.5) applying a multigrid approach, where the raw images were cross-correlated on a final interrogation window size of $24 \times 48 \mathrm{px}^{2}(y, z)$ with an overlap factor of $50 \%$. As such, the resulting velocity information was derived with a vector spacing of 0.2 and $0.4 \mathrm{~mm}$ in $y$ and $z$ directions, respectively.

\section{Results and discussion}

\subsection{Unforced flow}

Initially, no forcing was applied such to retrieve a measurement of the naturally-evolving channel flow (referred to as the reference measurements) and in order to verify the quality and performance of the sPIV 
system. Various flow conditions $\left(3.7<U_{\mathrm{cl}}<10.6 \mathrm{~m} / \mathrm{s}\right)$ were tested and the evaluated friction velocity $u_{\tau}$ (see eq. 3) was used to compute the friction Reynolds number $\operatorname{Re}_{\tau}\left(=u_{\tau} h / \mathrm{v}\right)$ and both streamwise velocity profiles $U^{+}\left(y^{+}\right)$and Reynolds-stress profiles of the time- and spanwise-averaged velocity fields. Since the spatial resolution was not sufficient, for directly computing $u_{\tau}$ from the velocity gradient $\mathrm{d} U / \mathrm{d} y$ in the viscous sublayer $\left(y^{+} \leq 5\right)$, the estimation of $u_{\tau}$ was performed both by a least-sqaure fitting of the analytical form for the mean-velocity profile, provided by Luchini (2018), with the actual velocity profile and by the computation of the total stress (Pope (2000))

$$
\tau_{x y}=\mu \frac{\mathrm{d} U}{\mathrm{~d} y}-\rho \overline{u v} .
$$

The differences between both methods were less than $2.5 \%$. Since from the total stress in equation 2 the wall-shear stress $\tau_{\mathrm{w}}$ can also be computed, it is directly retrieved from the acquired velocity data. Therefore, the total-stress approach, as shown in figure 2 (a), was used to estimate $u_{\tau}$ by applying

$$
u_{\tau}=\sqrt{\frac{\tau_{\mathrm{w}}}{\rho}}
$$

to all flow conditions. In case of known wall position from the experiment, $\tau_{\mathrm{w}}$ results from a linear extrapolation of $\tau_{x y}$ (at $y=0$, i.e. at the wall). Hence, the required wall position, in this work, was extracted from the raw PIV images. As the inherent decrease of the total stress in the vicinity of the wall is unphysical and can be attributed to errors from both particle reflections and resolution limits of the sPIV, the linear extrapolation of $\tau_{x y}$ is based on a least-square fit (see fig. 2 (a), dashed line). For cases of unknown wall position, another robust approach that exploits the expression (Pope (2000))

$$
\tau_{x y}(y)=\tau_{\mathrm{w}}\left(1-\frac{y}{h}\right)
$$

can be used to compute $\tau_{w}$, by averaging the slope of the total stress $\tau_{x y}(y)$ along $y$. This procedure was also applied as a means of verification and culminated in less than $1 \%$ deviation from the results of the linear extrapolation.

(a)

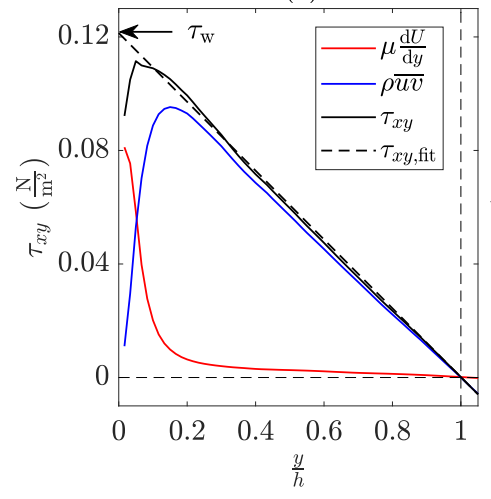

(b)

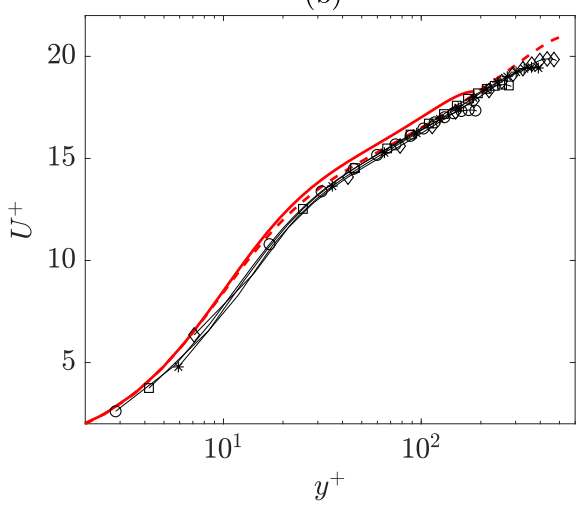

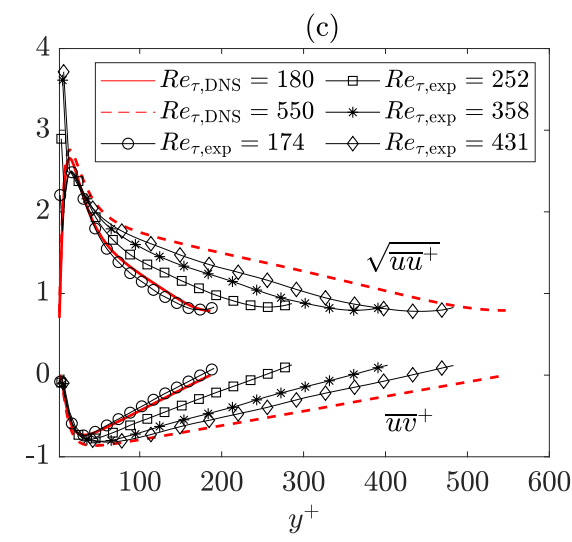

Figure 2: Comparison of sPIV results and DNS $\left(R e_{\tau}=180,550\right.$, Hoyas and Jiménez (2008)). (a) Evaluation procedure of $\tau_{\mathrm{w}}$ from the total stress. (b) Streamwise velocity profiles $U^{+}\left(y^{+}\right)$. (c) Root-mean-squared profiles $\sqrt{\overline{u u^{+}}}$and mean shear-stress profiles $\overline{u v}^{+}$.

The resultant streamwise velocity profiles $U^{+}\left(y^{+}\right)$(black lines) of the turbulent channel flow are shown in figure 2 (b), scaled in viscous units, for the tested range of Reynolds numbers $\left(174 \leq R e_{\tau} \leq 431\right)$. The comparison of the sPIV results and the direct numerical simulations (DNS) of Hoyas and Jiménez (2008) (red lines) depict a slight offset in the logarithmic region $\left(50<y^{+}<120\right.$, Pope (2000)), whereas in the viscous sublayer $\left(y^{+} \leq 5\right)$ the data points almost match the DNS. However, it is evident that the sublayer region was resolved by a single data point, for $R e_{\tau} \leq 252$, only. Therefore, the differences of the experimental data with respect to the DNS originate either from an overestimation of $u_{\tau}$ or a slightly increased friction drag caused 
by the surface structure of the actuator dielectric. For clarity, figure 2 (c) shows only the root-mean-squared profiles $\sqrt{\overline{u u^{+}}}$and the mean shear-stress profiles $\overline{u v}^{+}$that clearly indicate an excellent fit between the experimental results at the tested $R e_{\tau}$ range and the DNS. Typically, the uncertainty of PIV increases very close to the wall due to large velocity gradients in the turbulent boundary layer. This effect can be observed for $\sqrt{\overline{u u}^{+}}$for the near-wall data points. The peak at $y^{+} \approx 15$ is underestimated and even closer to the wall $\sqrt{\overline{u u}^{+}}$ reaches large values, diverging from the DNS data. The $\overline{u v}^{+}$component, instead, shows strong agreement with the DNS even in the near-wall region.

As an intermediate summary, the sPIV results have demonstrated that the compliance of the velocity data in viscous units, from estimation of $u_{\tau}$ by the total stress, and the DNS of Hoyas and Jiménez (2008) provide a solid basis for the second part in which topological interplay effects of oscillatory plasma discharges and channel flow will be investigated. It is further to be noted that the scope in this part was to create a reliable experimental framework that allows for comparisons of uncontrolled and controlled flows which was accomplished by assessing the deviations of the experimental data set and the DNS reference.

\subsection{Forced flow}

In previous studies, it has been demonstrated that plasma actuators can be driven such to generate oscillating plasma discharges (Wilkinson (2003), Jukes et al. (2006)), in order to mimic spanwise wall oscillations. One of the electrode arrays featured the configuration of the exposed (light gray) and encapsulated (dark gray) electrodes in the bottom part of figure 3 (Jukes et al. (2006)), neglecting the red electrodes. The discharge is generated above the encapsulated electrodes (dark gray) and induces a flow that periodically changes direction. As a drawback in terms of spanwise flow homogeneity, no discharge is generated in the gaps (positions of red electrodes). Therefore, further encapsulated electrodes (red) were added, achieving an array of side-by-side arranged exposed (light gray) and encapsulated (red and dark gray) electrodes. Hence, formerly existing gaps of no discharge Jukes et al. (2006) were closed and, at the same time, minimized to the width of the exposed electrodes. This particular electrode configuration, as developed by Hehner et al. $(2019,2020)$, was applied in the present work.

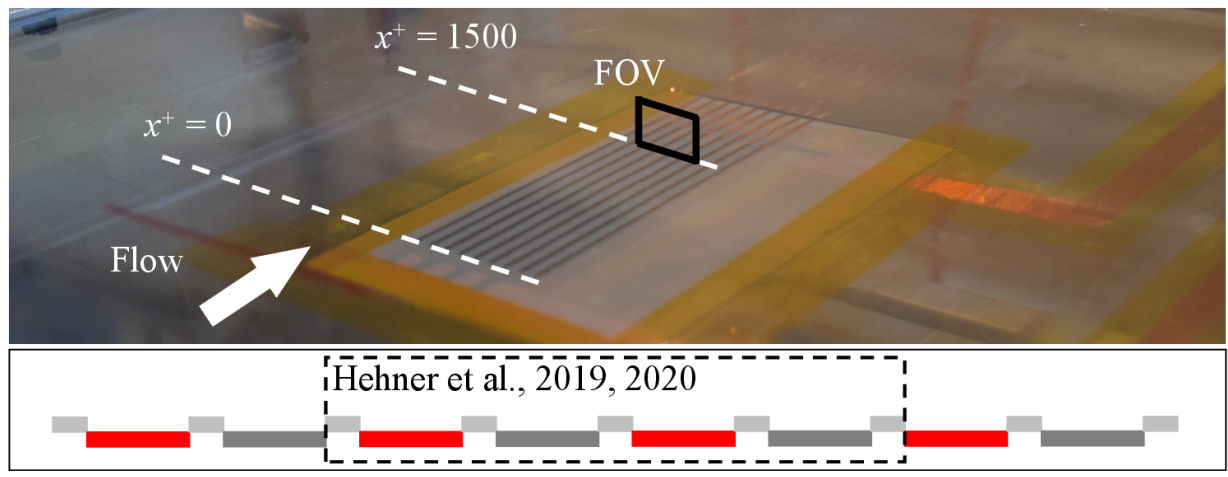

Figure 3: Flush-mounted plasma actuator for spanwise oscillating discharges. Positions $x^{+}=0$ and 1500 for $R e_{\tau}=252$, FOV and flow direction are indicated. The sketch below shows a cross-section of the electrode configuration of the actuator (exposed electrodes: light gray; encapsulated electrodes: dark gray and red).

The full plasma-actuator array consists of 9 exposed and 8 encapsulated electrodes of 1 and 3 mm width, respectively (spanwise wavelength $\lambda_{z}=4 \mathrm{~mm}$ ). The dielectric material was a polyester foil (Mylar®A, $500 \mu \mathrm{m}$ thickness) and the electrodes consisted of silver ink (Silverjet DGP-40LT-15C, Sigma-Aldrich ${ }^{\circledR}$ ) applied to the dielectric by a fully-automated inkjet-printing method (printer model: Autodrop Professional by microdrop Technologies $\mathrm{GmbH}$ ), resulting in an electrode thickness of $<1 \mu \mathrm{m}$. The actuator was flushmounted on the surrounding substrate, by means of a cutout of a polyethylenterephthalate foil of equal thickness as the dielectric (see fig. 3).

In two recent works, this type of plasma actuator was operated either in burst-modulation (Hehner et al. (2019)) or beat-frequency mode (Hehner et al. (2020)) and the underlying results from quiescent air have confirmed its capability to impose an oscillatory spanwise fluid motion on the surrounding air. Due to the non-linear nature of the forcing with plasma, modifying the flow and the flow influencing the forcing, a superposition of plasma-induced flow in quiescent air and channel flow is prohibitive. Yet, this arises 
the question for the inherent interplay effects of external flow on the plasma-induced flow topology and vice versa. Therefore, and as a next logical step, the measurements were now performed both in quiescent air and in the channel flow when the plasma actuator was switched on in beat-frequency mode.
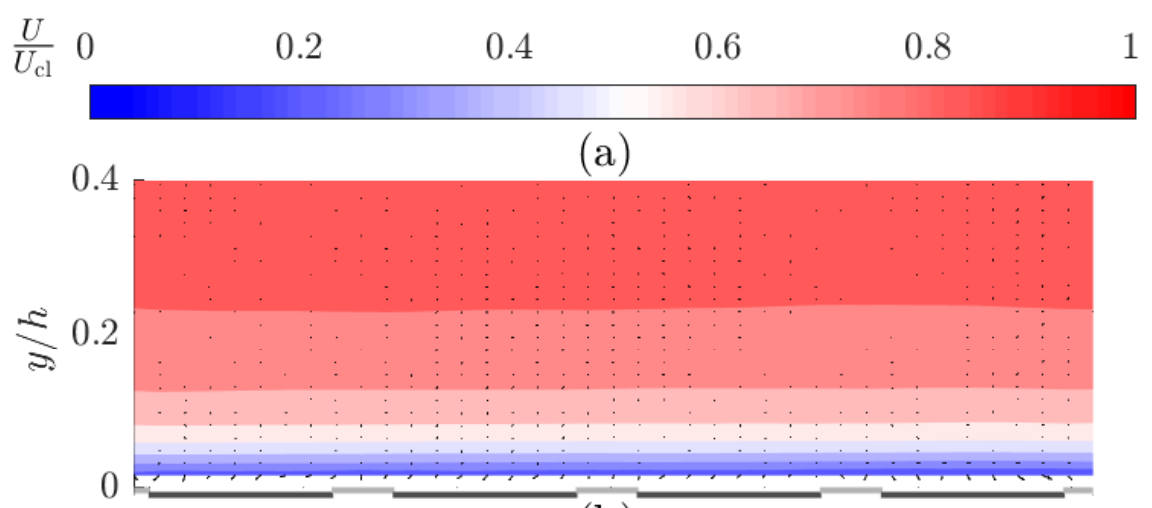

(b)

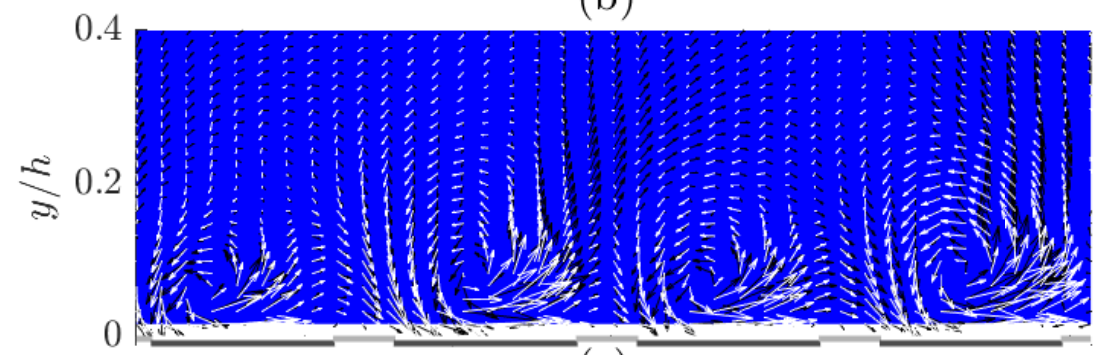

(c)

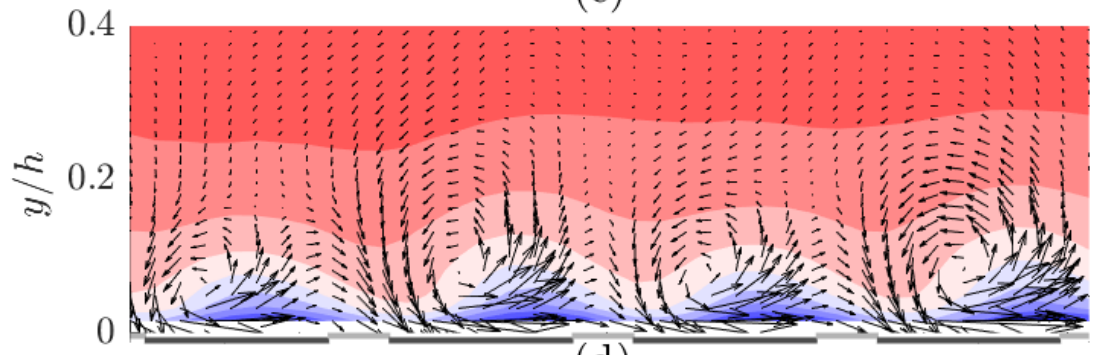

(d)

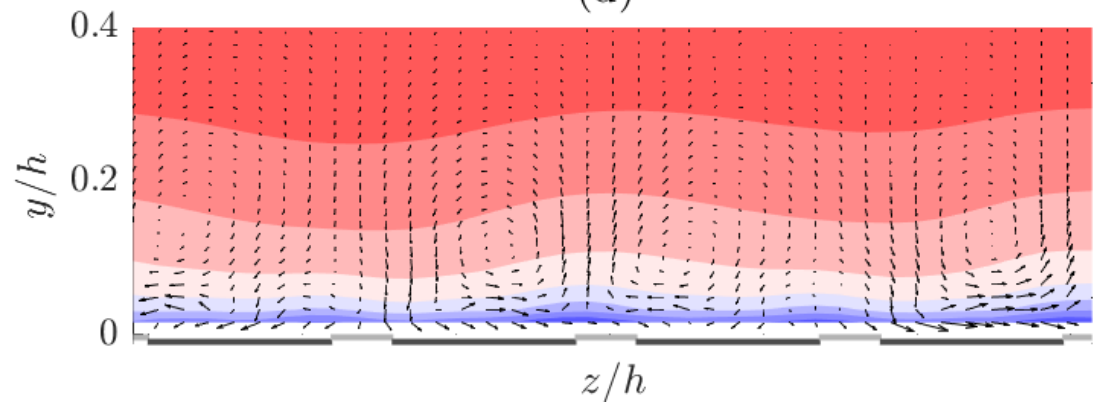

Figure 4: Velocity contours of $U / U_{\mathrm{cl}}\left(U_{\mathrm{cl}}=5.86 \mathrm{~m} / \mathrm{s}, R e_{\tau}=252\right)$ and velocity vectors $(v, w)$. (a) Timeaveraged non-actuated flow at $R e_{\tau}=252$. (b), (c) Phase-averaged $(\Delta \varphi=5 / 6 \pi$ after discharge onset) flow topology for actuation in quiescent air and at $R e_{\tau}=252$, respectively. The white vectors in (b) were copied from (c) for comparison with the induced flow in quiescent-air conditions. (d) Time-averaged flow topology for actuation at $R e_{\tau}=252$. The exposed and encapsulated electrodes are indicated by the light and dark gray rectangles, respectively.

The case of $R e_{\tau} \approx 250$, anticipated in Hehner et al. (2019,2020), was selected. Accordingly, the operating parameters were such to generate a spanwise oscillation at frequency $f=50 \mathrm{~Hz}$ and a plasma frequency $f_{\mathrm{ac}}=16 \mathrm{kHz}$. The streamwise length of the plasma actuator was $x^{+}=1620$, where $x^{+}=0$ refers to the 
upstream edge of the plasma. The measurements were conducted at $x^{+} 1500$ (see also fig. 3), near the downstream edge of the actuator.

The style of figure 4 is such that the out-of-plane component $U / U_{\mathrm{cl}}\left(U_{\mathrm{cl}}=5.86 \mathrm{~m} / \mathrm{s}\right)$ is shown as velocity contours, the velocity vectors of the in-plane components $(v, w)$ are superimposed and the electrodes are indicated by the gray rectangles below the fields. The time-averaged velocity field of the uncontrolled reference flow at $x^{+}=1500$ is shown in figure 4 (a). No modulation effect from the surface irregularities, due the electrodes, on the streamwise flow component is detected. It is further to be noted that the results of figure 2 also refer to $x^{+}=1500$.

For the plasma cases phase-resolved data was recorded and divided into 24 bins of the oscillation cycle (phase difference of consecutive snapshots $\Delta \varphi=\pi / 12$ ). Hence, the velocity field in quiescent air as an average of a single phase is shown in figure 4 (b). The typical inhomogeneity of the the spanwise flow due to discharge gaps of the exposed electrodes can be observed (Hehner et al. $(2019,2020)$ ). As an important aspect, the reconstructed out-of-plane component $U / U_{\mathrm{cl}}$ is identical to zero. The velocity field of the same phase position as in figure 4 (b) is displayed in figure 4 (c), when the windtunnel was turned on at set to cause a center-line velocity of about $5.86 \mathrm{~m} / \mathrm{s}$. The plotted velocity vectors were copied and added to figure 4 (b) in white, in order to allow for a better comparison of the non-linear effect of the channel flow on the plasma-induced flow topology. At first glance, the differences appear minor, however, there is a decrease of the velocity magnitude of the in-plane components at $0.2<y / h<0.4$ and in the near-wall region on the side of the large velocity components some vectors occur at a more shallow angle as compared to quiescent air. The plasma actuation, on the other hand, has significant effect on the channel flow which is expressed by a spanwise modulation of the streamwise flow component which occurs at $\lambda_{z}$ of the plasma actuator. The time-averaged topological effect of the plasma actuation on the channel flow is shown in figure 4 (d). As a difference to figure 4 (c), the length of the wave-like spanwise modulation of $U / U_{\mathrm{cl}}$ is increased. While in the near-wall region (blue contours) the footprint of the modulation at $\lambda_{z}$ can be retrieved, the time-averaged velocity vectors show weak vortical structures in the center and on the sides of the velocity field. This is supposed to be a consequence of slight differences in the induced velocity magnitudes along the oscillation cycle of the plasma discharge.

\section{Concluding remarks}

The present work has verified the suitability of the chosen sPIV setup used to investigate a fully-developed turbulent channel flow and to study the interplay effects of external flow and plasma discharges. The total stress has been computed to evaluate $\tau_{\mathrm{w}}$ and estimate $u_{\tau}$, showing very good agreement with the DNS of Hoyas and Jiménez (2008). The drawback of the sPIV setup is the resolution limit that hinders a direct evaluation of $u_{\tau}$ from the velocity gradient $\mathrm{d} U / \mathrm{d} y$ in the viscous sublayer $\left(y^{+} \leq 5\right)$. The comparison of plasma actuation in quiescent air and in a channel flow yields weak differences of the in-plane velocity fields $(v, w)$, whereas a significant effect on the turbulent boundary layer, exhibiting a spanwise modulation, was achieved.

\section{Acknowledgements}

The authors acknowledge the provision of lab equipment from the research group of Marios Kotsonis at TU Delft.

\section{References}

Auteri F, Baron A, Belan M, Campanardi G, and Quadrio M (2010) Experimental assessment of drag reduction by traveling waves in a turbulent pipe flow. Physics of Fluids 22:115103

Benard N and Moreau E (2014) Electrical and mechanical characteristics of surface ac dielectric barrier discharge plasma actuators applied to airflow control. Experiments in Fluids 55:1-43

Choi KS, DeBisschop JR, and Clayton BR (1998) Turbulent boundary-layer control by means of spanwisewall oscillation. AIAA journal 36:1157-1163 
Gatti D and Quadrio M (2016) Reynolds-number dependence of turbulent skin-friction drag reduction induced by spanwise forcing. Journal of Fluid Mechanics 802:553-582

Hehner M, Gatti D, Mattern P, Kotsonis M, and Kriegseis J (2020) Beat-frequency-operated dielectricbarrier discharge plasma actuators for virtual wall oscillations. AIAA Journal 59:1-5

Hehner MT, Gatti D, and Kriegseis J (2019) Stokes-layer formation under absence of moving parts-a novel oscillatory plasma actuator design for turbulent drag reduction. Physics of Fluids 31:051701

Hoyas S and Jiménez J (2008) Reynolds number effects on the reynolds-stress budgets in turbulent channels. Physics of Fluids 20:101511

Jukes T, Choi KS, Johnson G, and Scott S (2006) Turbulent drag reduction by surface plasma through spanwise flow oscillation. in 3rd AIAA Flow Control Conference. page 3693

Kotsonis M (2015) Diagnostics for characterisation of plasma actuators. Measurement Science and Technology 26:092001

Kriegseis J, Mattern P, Nawroth G, Vaas M, and Frohnapfel B (2015) Coherent secondary flow patterns in turbulent open duct flow. 11th International Symposium on Particle Image Velocimetry - PIV15, Santa Barbara, CA, USA

Kriegseis J, Simon B, and Grundmann S (2016) Towards in-flight applications? a review on dielectric barrier discharge-based boundary-layer control. Applied Mechanics Reviews 68:020802

Luchini P (2018) Structure and interpolation of the turbulent velocity profile in parallel flow. European Journal of Mechanics-B/Fluids 71:15-34

Pope SB (2000) Turbulent Flows. Cambridge University Press

Quadrio M (2011) Drag reduction in turbulent boundary layers by in-plane wall motion. Philosophical Transactions of the Royal Society A: Mathematical, Physical and Engineering Sciences 369:1428-1442

Quadrio M and Ricco P (2011) The laminar generalized stokes layer and turbulent drag reduction. Journal of Fluid Mechanics 667:135-157

Wilkinson SP (2003) Investigation of an oscillating surface plasma for turbulent drag reduction. 41st Aerospace Sciences Meeting \& Exhibit 